\title{
COMMENT
}

DOI: $10.1057 / s 41599-017-0039-7$

\section{Should we discipline interdisciplinarity?}

\author{
Gabriele Bammer ${ }^{1}$
}

\begin{abstract}
Could we overcome the challenges of embedding interdisciplinarity in the academic mainstream if relevant expertise were defined and recognised as a new discipline? Such expertise includes the ability to combine knowledge from different disciplines, as well as to assess which disciplines and stakeholders have relevant perspectives, figure out how elements of problems are interconnected, decide how to deal with critical unknowns, and use research to support evidence-based change. A new discipline of integration and implementation sciences (I2S) would codify such knowledge and skills, especially for dealing with complex societal and environmental problems. It would operate in an analogous way to the discipline of statistics, as a: (1) collaborative hub within teams, (2) focus for dedicated methods journals and (3) lobby group for effective application. Key initial tasks are to develop a repository of currently hard-to-access methods, and an identifiable academic community; both guided by a unifying and motivating vision of where disciplining interdisciplinarity will lead.
\end{abstract}

\footnotetext{
${ }^{1}$ National Centre for Epidemiology and Population Health, Research School of Population Health, The Australian National University, 62 Mills Road, Acton, ACT 2601, Australia. Correspondence and requests for materials should be addressed to G.B. (email: Gabriele.Bammer@anu.edu.au)
} 


\section{Background}

nterdisciplinary researchers are hard to embed in the academic mainstream, despite growing recognition of their importance and increasing numbers of interdisciplinary projects and programmes (Ledford, 2015). There are long-standing issues with inadequate assessment mechanisms for tenure and promotion (National Academy of Sciences, National Academy of Engineering \& Institute of Medicine, 2005; British Academy Working Group on Interdisciplinarity, 2016; Klein and Falk-Krzesinski, 2017) and lower levels of success in grant applications (Bromham et al., 2016; Reckling and Fischer, 2010). This prompts two key questions:

- what is it about interdisciplinarity that does not fit in the academic mainstream?

- what needs to change: interdisciplinarity, the academic mainstream or both?

These questions have only been addressed in a piecemeal fashion, with most focus on changing the academic mainstream, especially moving away from its disciplinary foundations. Here I add a new consideration: changing interdisciplinarity, specifically the team-based interdisciplinarity that is essential for better understanding and acting on complex societal and environmental problems-such as reducing the gap between rich and poor, combating illicit drug use, controlling spiralling health care costs, and achieving sustainable socio-ecological systems.

Team-based interdisciplinarity addressing complex societal and environmental problems needs specific expertise over and above that contributed by disciplines. This set of knowledge and skills is currently poorly defined and recognised. If contributing such know-how was an established role, it could provide a way of more adequately integrating interdisciplinary researchers into academic institutions. Furthermore, the time is ripe to codify that expertise by pulling together lessons from decades of experience.

To illustrate what is needed, let us look more closely at research on illicit drug use as an example of a complex problem. Each relevant discipline brings an important, but only a partial, understanding to bear. For example, pharmacologists contribute knowledge about the effects of these drugs, epidemiologists about estimated levels of use in the population, criminologists about impacts on property theft and other crime, legal experts about regulations and laws, historians about how those laws came into being, and so on.

But it is no existing discipline's business to combine these disciplinary perspectives to allow illicit drug use to be viewed more comprehensively. Not only does this process of combination require a solid theoretical and methods base, it also requires consideration of a range of associated issues, including:

- which disciplines have useful knowledge to contribute

- which stakeholder perspectives would be valuable, such as from police and drug users

- whether and how different elements of the problem are interconnected, such as examining all the impacts of criminalizing drug use (including on deterrence, punishment, willingness to seek help, and reintegration into paid employment)

- the likely consequences of critical unknowns for understanding illicit drug use (such as rates and causes of cessation) or for changing illicit drug policy (such as the potential impact of a yetto-be developed synthetic drug or an unforeseen change in popular culture which alters perceptions about illicit drug use)

- how research can best support evidence-based change.

Particularly significant is that the relevant theory and methods can be used for a wide range of problems, not just illicit drug use.

To further develop this argument, let us narrow the focus to methods alone. The aim is not to denigrate or dismiss the importance of theory, nor the epistemological and ontological foundations of methods. Instead it is to show that even the narrower focus on methods alone demonstrates the requirement for a substantial level of expertise beyond that encompassed by the established disciplines.

For methods alone, the challenges for recognising interdisciplinary expertise are that:

1. seemingly unrelated techniques (e.g., for knowledge synthesis and managing unknowns) are not recognised as part of an interconnected set of methods that allow complex problems to be addressed more comprehensively

2. information about relevant methods is widely dispersed throughout the literature, making them hard to access

3. there is no formal academic acknowledgement for developing, compiling, applying and promoting such methods.

What if we established a new discipline to underpin teambased interdisciplinary research on complex societal and environmental problems? A discipline that sets out an organised approach to dealing more comprehensively with such problems? One aspect of such a discipline would be to provide a repository for the methods that are currently no other discipline's business. These new disciplinary experts would then join teams tackling complex problems to-among other things-make them aware of, and help them apply, the best available methods.

A discipline of integration and implementation sciences (I2S) has been proposed to fill such a role (Bammer, 2013). As currently proposed, the I2S framework organises methods into three domains:

1. synthesis of disciplinary and stakeholder knowledge

2. understanding and managing critical unknowns

3. providing integrated research support (bringing together both what is known and an approach to critical unknowns) for policy and practice change.

A discipline is, of course, more than a repository of methods. Nevertheless, the benefits of such a repository are a primary focus here. A second focus is the political role of a discipline in providing an identity for an academic community: those who build the discipline, promote agreed standards, train the next generation, and advocate in the research and education policy and funding worlds.

\section{Integration and implementation sciences (I2S) in operation}

The discipline of statistics provides three productive analogies both for how I2S would work and for overcoming major challenges to getting there. Key to success in both are: collaboration, dedicated methods journals, and lobbying for effective application of the discipline.

Collaboration. For both statistics and I2S to be effective, collaboration is essential. Both enhance research across a wide range of problem areas-health, the environment, education and more. Statisticians contribute to teams that require expertise in quantitative reasoning. They assist teams in designing studies and in interpreting the outcomes.

Integration and implementation scientists would contribute to teams requiring expertise in exploring a complex problem more comprehensively. They would assist teams in figuring out how best to approach the problem and its interconnections, which disciplines and stakeholders need to be involved, how to bring together the various disciplinary and stakeholder perspectives, how to take into account what's not known about the problem, how to support those charged with acting on the problem, and other related issues.

To be more specific, for the illicit drugs problem described above, integration and implementation scientists would be able to 
advise, for example, on different approaches to combining knowledge from disciplines and stakeholders-such as dialogue, modelling or developing a joint vision-along with a range of formal and informal methods for undertaking each of these, such as nominal group technique (Delbecq et al., 1975), system dynamics modelling (Sterman, 2000) and appreciative enquiry (Cooperrider and Whitney, 2005).

Dedicated methods journals. If a statistician's work in a project team leads to advances in the statistical methods they brought to the table, the innovation is reported in the statistics disciplinary literature, not in the literature about the problem they were working on. The advance then becomes available to all other statisticians to apply, as appropriate, in the full range of problem areas, be they in education, environment, security, health or elsewhere.

I2S needs similar journals to share relevant techniques among researchers undertaking interdisciplinary explorations of various complex problems. If an integration and implementation scientist in a team working on illicit drug use developed an innovative scoping technique to identify all the relevant disciplines and stakeholders, for instance, there is currently no journal through which this advance could be made available to others tackling, for example, biodiversity loss, obesity, or poverty reduction.

Instead, relevant methods tend to be published in the literature about the societal or environmental problem, in the grey literature, or not published at all. As well as making methods hard to find, this means that any research team's knowledge about the methods that are already available is poor, leading to a lot of reinventing of methods for, for instance, engaging stakeholders and translating evidence for practitioners. It also means that existing methods tend to stagnate rather than being continuously improved.

Lobbying for effective application of the discipline. The importance of collaboration means that it is not enough for statistics and I2S to simply exist; each needs to be effectively brought into the relevant partnerships. In recent years, statisticians have banded together to ensure that statistical understanding and tools are appropriately deployed. For example, because analysis cannot fix poor design, they have effectively lobbied and educated for inclusion of statisticians at project start-up, rather than the previous practice of just bringing them in at the later analysis stage. As a result, the statistical approaches in grant applications and papers now come under close scrutiny, with funders and journal editors often requesting specific reviews by expert statisticians.

Similarly, it will not be enough for I2S just to be established; action will also be required to ensure that integration and implementation scientists have influence in research on complex societal and environmental problems. The role of I2S is to raise the bar in teams tackling these issues. An immediate task is to move teams away from reinventing methods to employing and building on those that already exist.

A major hurdle to promoting such adoption of I2S is again fragmentation - this time the lack of a unified academic community to drive uptake of I2S.

There are two separate fragmented groups of researchers who need to unite in common cause. First are the so-called "T-shaped researchers" (Brown et al., 2015) from teams working on complex societal and environmental problems. The name recognises not only their skills in a traditional discipline (the vertical bar), but also their ability to collaborate across disciplines (the horizontal bar). Nevertheless, there is no unified community of T-shaped researchers sharing and promoting these "horizontal bar" skills.

Second, there are researchers who have sought to develop intellectual agendas and professional associations around ways of more comprehensively viewing and acting on complex problems.
Examples of the critically important work undertaken by these researchers can be found in a wide spread of literatures, including those in interdisciplinary research (e.g., Frodeman et al., 2017; Repko and Szostak, 2016), transdisciplinary research (e.g., Hirsch Hadorn et al., 2008; Bergmann et al., 2010), systemic intervention (e.g., Midgley, 2000), system dynamics (e.g., Sterman, 2000), community operational research (e.g., Johnson, 2011), complex systems science (e.g., Mitchell, 2009), sustainability science (e.g., Clark and Dickson, 2003), action research (e.g., Bradbury, 2015), and change management (e.g., Nauheimer, 1997). Although many of the insights, theory and methods developed by researchers in these areas overlap, there are no existing mechanisms to link them. Indeed the development of a new 'interdisciplinary' discipline would draw together and build on this work.

Some of these intellectual pursuits have led to the development of professional associations; others have led to the formation of networks or other communities of practice. Here I focus on professional associations, which tend to be small-with membership in the hundreds rather than thousands-and they have few intersections, despite their common interests. Examples include the Association for Interdisciplinary Studies (https://oakland.edu/ais/), the International Society for Systems Sciences (http://isss.org/ world/) and the Action Learning, Action Research Association Inc (https://www.alarassociation.org/). Their small memberships make it hard for them to speak with authority in policy discussions about funding, research or education. Further, many of the T-shaped researchers are not members of these existing associations and may, indeed, not even know about them.

The aim of I2S is to provide a unifying focus and rationale for banding together.

\section{Moving forward}

Two pressing tasks for I2S are to overcome the fragmentation of methods and of academic communities, through building a methods repository and providing an identity, both of which are primary missions of disciplines. The aim of an I2S discipline identity is not to replace or subsume the existing interdisciplinary, systems thinking, action research and other such professional associations, but-by providing a common identity around integration and implementation expertise- to provide a conduit connecting them.

Building the methods repository is a way to make that conduit real. It requires:

- scouring the work of teams and professional associations to find all the relevant methods

- fostering collaboration among those identifying as integration and implementation scientists to develop consensus on

o which methods should be included

o how they should be catalogued

- facilitating discussion about epistemological and ontological differences in the foundations of the methods and what those mean for the repository

- generating evidence to match methods to the particular circumstances of a research investigation, such as which dialogue tools are most effective for marginalised stakeholder groups or which engagement tools work best for policy makers.

The Integration and Implementation Insights blog (http:// I2Insights.org) has started this process. It contains well over 150 descriptions of relevant methods and concepts contributed by more than 180 authors from 24 countries, with readers from over 185 countries.

To gain traction I2S needs to provide not only a repository, identity and conduit connecting teams and professional 
associations, but also a unifying and motivating vision for where disciplining interdisciplinarity would lead.

My vision starts with multiple interdisciplinary research teams - such as a team project on fisheries depletion in Sweden, a government research team investigating firearms control in Brazil, a graduate student programme tackling obesity in the USA, and multiple research groups in a public-private partnership addressing poverty in sub-Saharan Africa. At least one member of each team is an integration and implementation scientist, and belongs to a vibrant international academic community striving for continuous improvement in ways to tackle complex societal and environmental problems. Their work has a strong basis in theory and methods.

On the methods front, through text books, journals, and an open-access online methods bank, they have access to, and help upgrade, a repository of methods for dealing more comprehensively with complex problems. They provide their teams with practical assistance in choosing and applying the best methods for their investigations, and use that experience to evaluate and improve methods. Their performance for tenure and promotion is assessed in an analogous way to that of statisticians: including by the methods they have developed and improved and by their ability to contribute to the team in progressing understanding and action on complex societal and environmental problems; all evidenced through publications and grants. Critically, the assessment is conducted by peer integration and implementation scientists (Bammer, 2016).

Disciplining interdisciplinarity could provide a break-through in the way interdisciplinary researchers in teams addressing complex societal and environmental problems are embedded in the academic mainstream. It would recognise that traditional disciplinary expertise must be complemented by specialist skills to weave disciplinary and other insights into a more comprehensive understanding of the problem as a whole, as well as providing effective options for action.

Developing such a discipline requires considerable further work, including agreeing on the core knowledge and skills required to be an integration and implementation scientist-not only in methods, but also in theory-as well as systematically laying out the existing knowledge that will provide the foundations for the discipline and examining how the notion of a discipline fits into the diverse debates about interdisciplinarity. It will also require political action to advance the status and strength of the discipline, for example, by recognising windows of opportunity in changing research and education policy and using them to cement a unifying identity.

The payoff both for interdisciplinary researchers and for more effective understanding of, and action on, complex societal and environmental problems promises to be great.

Received: 6 August 2017 Accepted: 30 October 2017

Published online: 14 November 2017

\section{References}

Bammer G (2013) Disciplining interdisciplinarity: Integration and implementation sciences for researching complex real-world problems. ANU Press, Canberra, http://press.anu.edu.au?p=222171. See also the Integration and Implementation Sciences website http://i2s.anu.edu.au

Bammer G (2016) What constitutes appropriate peer review for interdisciplinary research? Palgrave Communications 2, 16017 (2016). https://doi.org/10.1057/ palcomms.2016.17

Bergmann M, Jahn T, Knobloch T, Krohn W, Pohl C, Schramm E (2010) Methoden transdisziplinaerer Forschung: Ein Ueberblick mit Anwendungs beispielen. Campus Verlag, Frankfurt, English version published in 2012: Methods for transdisciplinary research. A primer for practice

Bradbury H (ed) (2015) The Sage handbook of action research, 3rd edn. Sage, Los Angeles
British Academy Working Group on Interdisciplinarity (2016) Crossing paths: Interdisciplinary institutions, careers, education and applications. The British Academy for the Humanities and Social Sciences, London

Bromham L, Dinnage R, Hua X (2016) Interdisciplinary research has consistently lower funding success. Nature 534(30 June):684-687

Brown RR, Deletic A, Wong THF (2015) How to catalyse collaboration. Nature 525:315-317

Clark WC, Dickson NM (2003) Sustainability science: the emerging research program. PNAS 100(14):8059-8061

Cooperrider DL, Whitney D (2005) Appreciative inquiry: a positive revolution in change. Berrett-Koehler, San Francisco

Delbecq AL, Van De Ven AH, Gustafson DH (1975) Group techniques for program planning: a guide to nominal groups and Delphi process. https://sites. google.com/a/umn.edu/avandeven/publications/books/group-techniques-forprogram-planning

Frodeman R (editor in chief), Klein JT, Pacheco RCS (associate editors) (2017) The Oxford handbook of interdisciplinarity, 2nd edn. Oxford University Press, Oxford

Hirsch Hadorn G, Hoffmann-Riem H, Biber-Klemm S, Grossenbacher-Mansuy W, Joye D, Pohl C, Wiesmann U, Zemp E (eds) (2008) Handbook of transdisciplinary research. Springer, New York

Johnson MP (ed) (2011) Community-based operations research: decision modeling for local impact and diverse populations. Springer, New York

Klein JT, Falk-Krzesinski HJ (2017) Interdisciplinary and collaborative work: framing promotion and tenure practices and policies. Research Policy 46 (6):1055-1061

Ledford H (2015) Team science or How to solve the world's biggest problems. Nature 525:308-311. http://www.nature.com/news/how-to-solve-the-worlds-biggest-problems-1.18367

Midgley G (2000) Systemic intervention: Philosophy, methodology, and practice. Kluwer Academic/Plenum Publishers, New York

Mitchell M (2009) Complexity: a guided tour. Oxford University Press, Oxford

National Academy of Sciences, National Academy of Engineering \& Institute of Medicine (2005) Facilitating interdisciplinary research. National Academies Press, Washington DC, https://doi.org/10.17226/11153

Nauheimer H (1997) The change management toolbook. A collection of tools, methods and strategies. http://www.change-management-toolbook.com/. Accessed 8 Nov 2017

Reckling FJ, Fischer C (2010) Factors influencing approval probability in Austrian Science Fund (FWF) decision-making procedures - FWF stand-alone projects programme, 1999 to 2008. SSRN (Social Science Research Network): https:// papers.ssrn.com/sol3/papers.cfm?abstract_id=1725985. Accessed 8 Nov 2017

Repko AF, Szostak R (2016) Interdisciplinary research: process and theory. Sage, Thousand Oaks, CA

Sterman JD (2000) Business dynamics. Systems thinking and modeling for a complex world. McGraw-Hill Higher Education, Boston

\section{Acknowledgements}

Valuable comments on drafts of this paper were received from Kenneth Baldwin, Lindell Bromham, Peter Deane, Alice Richardson (all from The Australian National University), Janet Hering (Swiss Federal Institute of Aquatic Science \& Technology; Eawag), Kirsten Kainz (University of North Carolina at Chapel Hill), Gerald Midgley (University of Hull), and Lisa Palmer (National Socio-Environmental Synthesis Center; SESYNC).

\section{Additional information}

Competing interests: The author declares no competing financial interests.

Reprints and permission information is available online at http://www.nature.com/ reprints

Publisher's note: Springer Nature remains neutral with regard to jurisdictional claims in published maps and institutional affiliations.

Open Access This article is licensed under a Creative Commons Attribution 4.0 International License, which permits use, sharing, adaptation, distribution and reproduction in any medium or format, as long as you give appropriate credit to the original author(s) and the source, provide a link to the Creative Commons license, and indicate if changes were made. The images or other third party material in this article are included in the article's Creative Commons license, unless indicated otherwise in a credit line to the material. If material is not included in the article's Creative Commons license and your intended use is not permitted by statutory regulation or exceeds the permitted use, you will need to obtain permission directly from the copyright holder. To view a copy of this license, visit http://creativecommons.org/ licenses/by/4.0/.

(C) The Author(s) 2017 